\title{
A Survey on Macro Environment Sensing using Sensors in Smart Devices
}

\author{
Nilesh Chavanke ${ }^{1}$, Harsimaran Saggu ${ }^{2}$, Sanket Kurude ${ }^{3}$, Saurabh Walunj ${ }^{4}$, Prof. S.D. Salunkhe ${ }^{5}$ \\ BE Student, Dept of IT, JSPM's Rajarshi Shahu College of Engineering, Tathawade, Pune, Maharashtra, India ${ }^{1,2,3,4}$ \\ Professor, Dept of IT, JSPM's Rajarshi Shahu College of Engineering, Tathawade, Pune, Maharashtra, India ${ }^{5}$
}

\begin{abstract}
A range of mobile and pervasive applications on recently smart phones. Although human-driven settings (e.g indoor/outdoor, at home/in work, driving walking) are wide researched. Proximity Sensing covering the various instances of phone use, situation, disposition, and cooperation altogether the approach right all the manner all the way down to earth utilizes with entangled shopper propensities. As an extended running middleware, Proximity Sensing considers every vitality utilization and shopper relationship. The preliminary results demonstrate that Proximity Sensing accomplishes low energy price, fast system preparation and competitive sensing accuracy.
\end{abstract}

Keywords: Proximity sensor, telephony manager, audio recognition system.

\section{INTRODUCTION}

There are many varieties of sensors as Proximity, measuring device, Camera, Touch, GPS etc. we tend to use these sensors associate degree developed Associate in nursing robot application. The conception of micro-environment sensing is created on each context sensing and context-awareness applications, however differs in its stress on perceiving immediate surroundings from the Smartphone's perspective. In mobile systems, context-awareness may possibly be a computing technology that options data regarding this atmosphere of a mobile user to supply further relevant services to the user.[1] It a key a locality of gift or pervasive computing and has attracted several analysis efforts at intervals the past decade. Mostcontext-aware applications (via movable sensing) are unit human-centric, recognizing contexts from users' perspective (e.g. indoor/outdoor, at home/in work, driving/walking. Such data supports services in line with users' state of affairs.

\section{LITERATURE REVIEW}

Noise Sense is required on the Apple iPhone and represents the most general purpose sound sensing system specifically designed to work on resource restricted phones. The look and algorithms area unit designed for quantify ability and noise Sense uses a combination of supervised and unproven learning techniques to reason every universal sound types (e.g., music, voice) and see work of fiction sound events specific to individual users. The system runs entirely on the changeable with no back-end connections.[2]

Mobile phones are becoming the convergent platform for personal sensing, computing, and communication. This paper makes a shot to require advantage of this convergence towards the matter of automatic image tagging. We tend to check Tag Sense, a mobile based totally cooperative system that senses the people, activity, associated context in an passing image, and merges them strictly to make tags on-the-fly. The foremost challenge pertains to discriminating phone users that as mobile apps become lots of closely integrated into our everyday lives, mobile app interactions have to be compelled to be quick and responsive. Sadly, even the elemental primitive of launching a mobile app is sorrowfully sluggish: twenty seconds of delay is not uncommon even for very standard apps. [5]

\section{PROPOSED SYSTEM}

Our application can be a unified framework covering the foremost very important cases of phone usage, placement, attitude, and interaction in wise uses with powerful user habits. The projected Proximity Sensing application accommodates six modules, initial module depends on the proximity detector, once the user switch-on the proximity detector provided in Proximity Sensing application if there is associate incoming call to the user then user would possibly simply pickup phone toreceive call or the user can simply inserting associate object around proximity detector. Second module depends on noise alert once user switch-on this in Proximity Sensing application it asks the user for threshold and any audio recognition system detects the noise of the peripheral atmosphere and if the detected noise exceeds the limit provided by user then the alert message is generated and sent to the registered mobile selection. Third module depends on shut atmosphere, once user ought to disable the screen of Smartphone to forestall unwanted touches 
the user would possibly switch-on this uses proximity detector for determinative unwanted touches done by user. Fourth module depends on bit pressure recorded on Smartphone that's supposed for providing security for ladies i.e. simply merely simply just in case of emergency the women can bit and hold her Smartphone for specific time then the alert message is distributed to the registered mobile selection with the GPS location therefore user is facilitate in emergency. If the un-authorized person accessing the user wise phone mechanically un-authorized person image is captured and current location is as well fetched and sent to user's mail id. Sixth model depends on the synchronizing the camera of user Smartphone with the system through Wi-Fi.

As associate degree extended running middleware, associate degree application considers each energy consumption and user friendly relationship. We have a developed associate degree application on automaton OS and consistently worth its performance with knowledge collected. The preliminary results show that associate degree application achieves low energy worth, speedy system preparation, and competitive sensing accuracy.The synchronizing the camera of user Smartphone with the system through Wi-Fi.

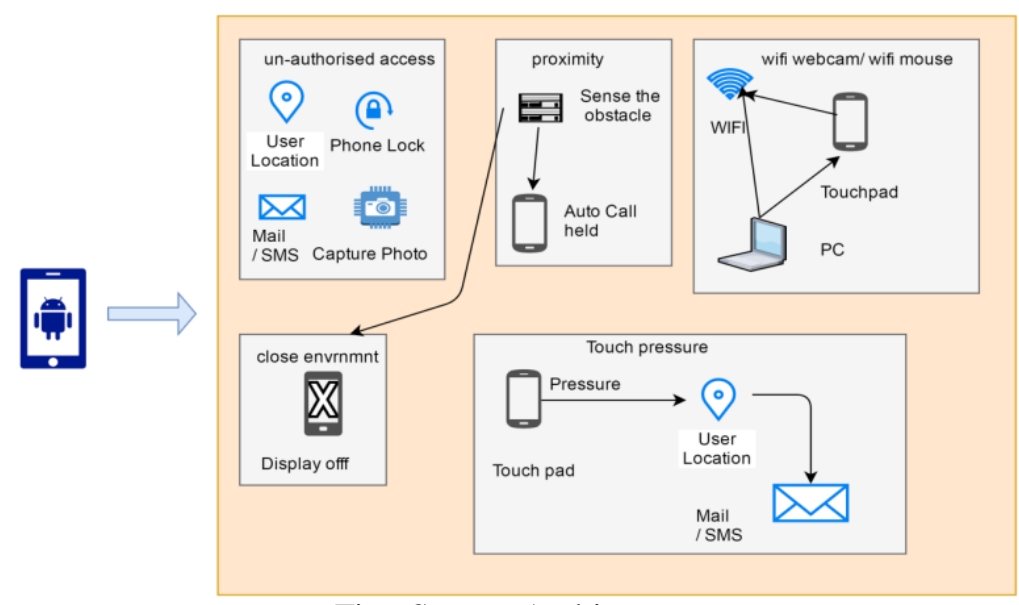

Fig.: System Architecture

\section{Automatic decision Picker:}

In this module we've a unit of activity reaching to use proximity device. We tend to face live planning to be checking open and shut conditions of proximity device. Suppose mobile is at intervals the pocket or in closed surroundings, then proximity device unit of measurement planning to be shut. Once carried by a user, the phone is usually placed in either semi-closed/open environments like in-hand, or closed environments like in-pocket and in-bag. The extent of covering ends up in completely different illuminative conditions for the phone, which could be captured by its inherent camera. Application mustn't receive square measure accessible therein purpose. We tend to unit of measurement planning to check Closeenvironment orOpenenvironments condition at that time. If mobile is in Open surroundings then we get the choice for Open condition of proximity device or we get the choice for close condition of proximity device.

\section{Pressure sensing element used for security:}

In this module, we use pressure detector of screen to measure the pressure on screen. If the pressure is larger than the brink pressure of application, then application will trigger the emergency message to emergency number which provided initially.

\section{Shut setting:}

In shut surroundings we have got associate degree inclination to tend to use the proximity device for this. Once the mobile is in shut surroundings at that if the choice is returning the screen unit of measurement near be OFF. If we tend to vary the setting that is close to open surroundings the screen unit of measurement near air.

\section{Noise Alert:}

We use the audio recognition system for recognizing the peripheral noise. If the audio recognized is larger than the brink then alert message unit of measurement getting to be generated and sent to the registered mobile number.

\section{Un-authorized Access:}

To prevent the user's Smartphone accessing from un-authorized person we created functionality captured image without knowing to unauthorised user. 


\section{Wi-Fi camera:}

User's Smartphone is connected to the system through Wi-Fi. The user sitting in-front of pc will see the image captured by the Smartphone.

\section{Third-Party provider Solutions:}

Last few years, an enormous vary of third-parties providing to deliver alert messages (and fully totally different knowledge services) via text electronic messaging services. The look of these systems is comparatively straightforward. Directly from a phone, or as package running on a field administrator's personal computer, these services act as SMS aggregators and inject text messages into the network.

\section{Connectify Me:}

Connectify Hotspot connects all devicesthrough wifi. This easy-to-use virtual router lets you share Internet from your laptop with your smartphone, tablet, media player, e-reader, and other laptops. Any wireless-equipped device can reach the Web through Connectify without any additional software. Connectify also encrypts the traffic to and from the software "hotspot" using WPA2-Personal (AES) encryption.

\section{Short Message Service:}

Short Message Service (SMS) may possibly be a text transmission service part of phone, web, or mobile communication systems, exploitation standardized communications protocols that modify the exchange of short text messages between mounted line and itinerant devices. SMS text transmission is that the foremost typically used data application at intervals the world, with 3.6 billion active users, or seventy eight of all itinerant subscribers. The term SMS is employed as constant word for all sorts of short text transmission to boot as a results of the user activity itself in several components of the globe. easy user generated text message services - embrace news, sport, financial, language and placement based mostly whole services, to boot as several early samples of mobile commerce like stocks and share costs, mobile banking facilities and leisure booking services. SMS has used on trendy handsets originated from radio telegraphy in radio memoranda pagers exploitation standardized phone protocols and later written as a vicinity of the world System for Mobile Communications (GSM) series of standards in 1985 as the simplest way of inflicting messages of up to 1 hundred sixty characters, to and from GSM mobile handsets. Since then, support for the service has expanded to incorporate numerous mobile technologies like ANSI CDMA networks and Digital AMPS, to boot as satellite and land line networks. Most SMS messages unit mobile-to-mobile text messages tho' the quality supports numerous sorts of broadcast transmission to boot.

\section{GSM Technology:}

GSM may possibly be a cellular network that suggests that cell phones connect with it by sorting out cells at intervals the immediate neighbourhood. There unit 5 fully totally different cell sizes in associate extremely GSM network. GSM uses several crypto logical algorithms for security. GSM network is sms.SMS purpose to purpose (SMS-PP) was originally made public in GSM recommendation that's presently maintained in 3GPP as defines the Short Message Service - Cell Broadcast (SMS-CB), that allows messages (advertising, public information, etc.) Messages unit of measurement sent to a short message service centre (SMSC) that has a "store and forward" mechanism. If the subscriber's mobile unit is powered off or has left the coverage house, the message is hold on and offered back to the subscriber once the mobile is powered on or has re-entered the coverage house of the network. This operate ensures that the message square measure progressing to be received.

\section{GPS Technology}

GPS might be a world navigation satellite system (GNSS) that has location and time information.The GPS system operates severally of any telecommunication or internet reception, though' these technologies will enhance the utility of the GPS positioning data. The GPS system provides essential positioning capabilities to military, civil, and industrial users round the world. the govt. created the system, makes it freely accessible to anyone with a GPS receiver. The GPS conception depends on time and jointly the celebrated position of specialized satellites. The satellites carry extremely stable atomic clocks that unit synchronous with each other and to ground clocks. Any drift from true time maintained on the lowest is corrected daily. Likewise, the satellite locations unit celebrated with nice accuracy. GPS receivers have clocks as well; but, they're typically not synchronous with true time, and unit less stable. A GPS receiver monitors multiple satellites and solves equations to examine the precise position of the receiver.

\section{Mathematical Model \\ Let ' $\mathrm{S}$ ' be the system \\ Where \\ $\mathrm{S}=\{\mathrm{I}, \mathrm{O}, \mathrm{P}\}$}


Where,

$\mathrm{I}=$ Set of input sensors

$\mathrm{O}=$ Set of output applications

$\mathrm{P}=$ Set of technical processes

Let ' $\mathrm{S}$ ' is the system

$\mathrm{S}=$ Identify the input sensors data $\mathrm{S} 1, \mathrm{~S} 2, \ldots ., \mathrm{Sn}$

$\mathrm{I}=\{$ Proximity, Accelerometer, Gyro, Pressure, Flash, GPS, Orientation $\}$

Identify the output applications as $\mathrm{O}$

$\mathrm{O}=\{$ Call picker, Location Traces, Closed environment identifier $\}$

Identify the Process as $\mathrm{P}$

$\mathrm{P}=\{$ Listener, Parser, Convertor, Logic Implementation $\}$

\section{RESULT ANALYSIS}

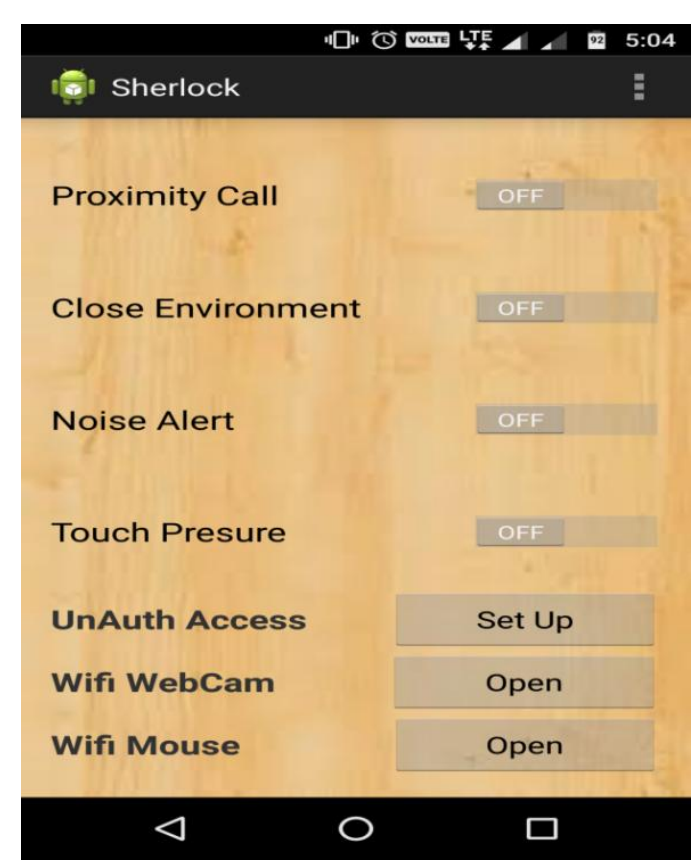

Main layout where we can enable or disable the services

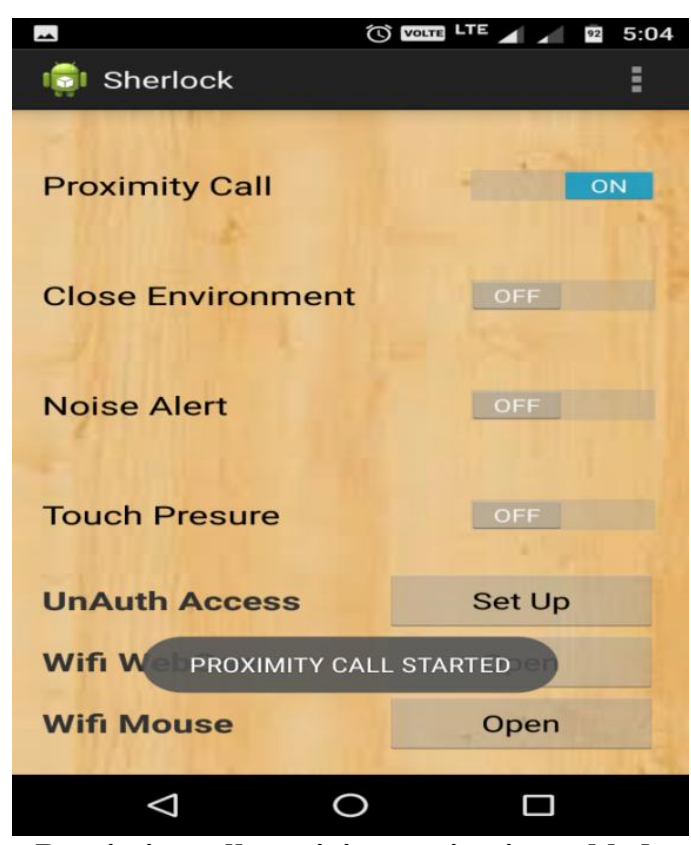

Proximity call receiving setting is enabled. 
International Journal of Advanced Research in Computer and Communication Engineering ISO 3297:2007 Certified

Vol. 6, Issue 5, May 2017

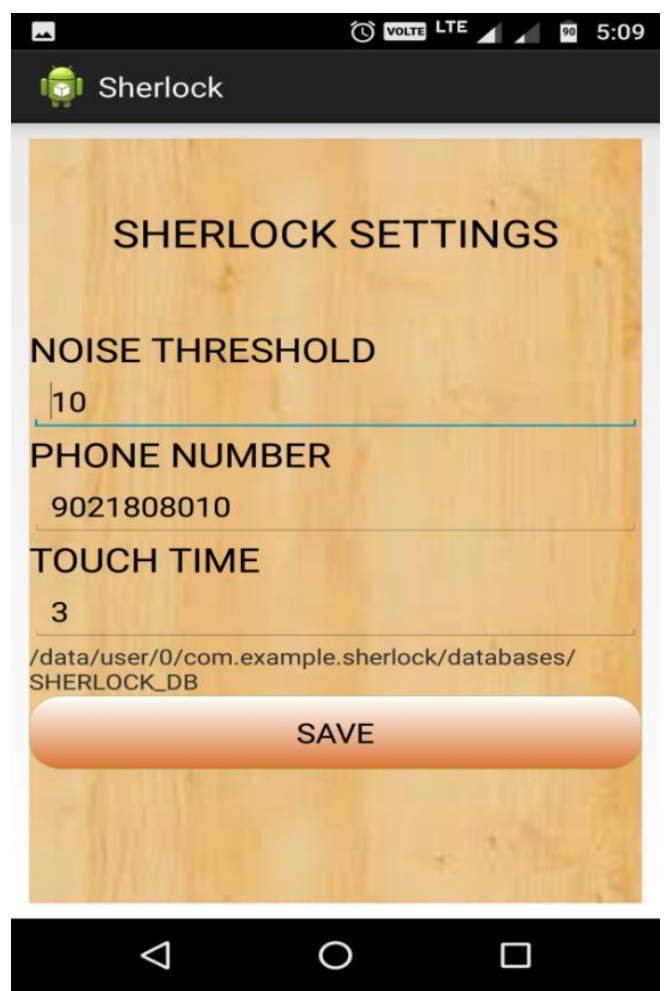

Start layout to get noise threshold, phone no, and touch time
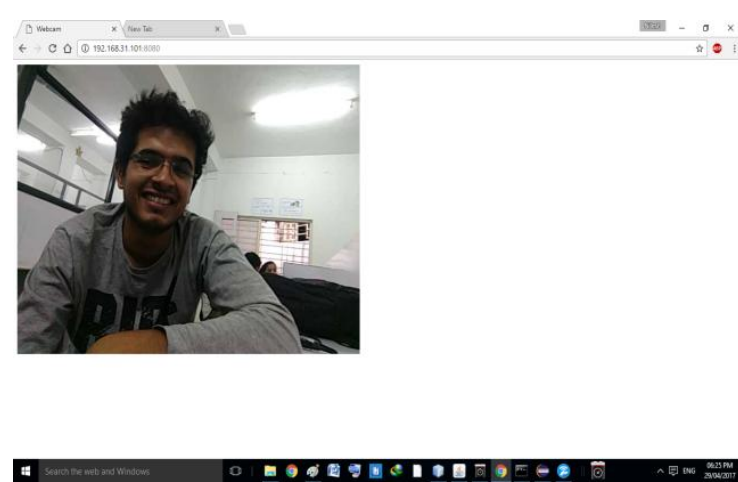

Wi-Fi Web cam module which display image on web browser

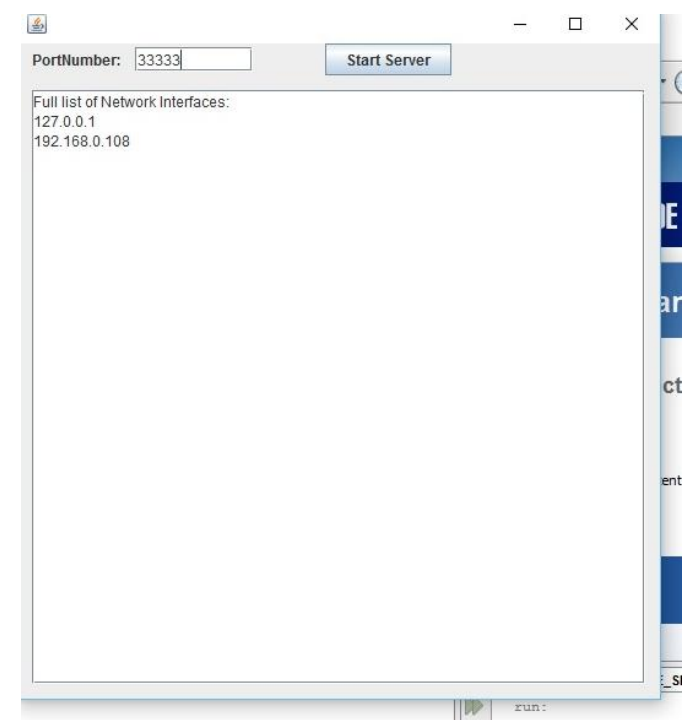

Wi-Fi mouse module: which suggest the list of IP to connect 


\section{FUTURE SCOPE}

There are really unlimited needs to sense the world and its processes. Unfortunately our own senses as well technical sensors are not perfect and error free. And even sensing something useful does not mean that the business around it makes value. These have the potential to collect data on us even when we are not actively using the phone. But most smartphones also have an image sensor, touch sensor, proximity sensor, and up to 30 other sensors, including GPS for location. New sensors are being developed all the time. Each one opens the door to new possibilities. A smartphone sensor that detects when food has gone bad .Imagine using your phone to check if the rotisserie chicken you brought home three days ago is still safe? Sensors make our phones more aware.

\section{CONCLUSION}

We gift the design, implementation and analysis supported android, a straightforward however good platform for microenvironment sensing for smartphones through collaboration among intrinsic sensors. The platform automatically collects sensing element hints and characterizes the immediate surroundings of smartphones at $\mathrm{cm}$ level accuracy, providing fine-grained atmosphere data to higher layer applications. We have got associate inclination to conduct comprehensive experiments to judge our system through an image implementation on android platform. Preliminary experiment results show that Proximity Sensing achieves low energy price, fast system preparation, and competitive sensing accuracy.

\section{ACKNOWLEDGMENT}

It is our privilege to express our sincerest regards to our project coordinator Prof S.D. Salunkhe for their valuable inputs, able guidance, encouragement, whole-hearted cooperation and constructive criticism throughout the duration of our project.

We deeply express our sincere thanks to our Head of Department Dr Prof. A.N. Banubakode for encouraging and allowing us to present the project on the topic "A Survey on Macro environment sensing using sensors in smart devices". We take this opportunity to thank all our lecturers who have directly or indirectly helped our project.

\section{REFERENCES}

[1] E. Y. Ahn, J. H. Lee, T. Mullen, and J. Yen, "Dynamic vision sensor camera based bare hand gesture recognition," in Proc. of the IEEE Symposium on Computational Intelligence for Multimedia, Signal and Vision Processing, 2011, pp. 52-59.

[2] C. Canali, G. D. Cicco, B. Morten, M. Prudenziati, and A. Taroni, "A temperature compensated ultrasonic sensor operating in air for distance and proximity measurements," IEEE Tran. Ind. Electron., vol. 29, no. 4, pp. 336-341, Nov. 1982.

[3] H. Cheng, A. M. Chen, A. Razdan, and E. Buller, "Contactless gesture recognition system using proximity sensors," in Proc. of the IEEE Int. Conf. on Consum. Electron., 2011, pp. 149-150.

[4] W. Chung, H. Kim, Y. Yoo, C. Moon, and J. Park, "The detection and followingofhumanlegsthroughinductiveapproachesforamobilerobot withasinglelaserrangefinder,"IEEETran.Ind.Electron.,vol.59,no.8, pp. 3156-3166, Aug. 2012.

[5] T. Delbruck and P. Lichtsteiner, "Photoarray for detecting timedependent image data," Patent US 20080135731 A1, $612,2008$.

[6] W. Hortschitz, H. Steiner, M. Sachsen, M. Sifter, F. Kohl, J. Schawk, A. Jachimowicz, F. Kiplinger, and T. Sauter,"Robust precision position detection with an optical mems hybrid device," IEEE Tran. Ind. Electron., vol. 59, no. 12, pp. 4855-4862, Dec. 2012.

[7] T. Inari and N. Aoki, "A proximity sensor using power variation of a laser diode caused by returned light and its characteristics of stability," in Proc. of the IEEE Annual Conf. on Ind. Electron., 2005, pp. 2114-2118.

[8] M. Jagiella, S. Feliciano, and A. Dorneich, "Progress and recent realizations of miniaturized inductive proximity sensors for automation," IEEE Sensors J., vol. 6, no. 6, pp. 1734-1741, Dec. 2006

[9] S. J. Kim and B. K. Kim, "Dynamic ultrasonic hybrid localization system for indoor mobile robots," IEEE Tran. Ind. Electron., vol. 60, no. 10, pp. 4562-4573, Oct. 2013. [10] Y. K. Kim, Y. Kim, Y. S. Jung, I. G. Jang, K. Kim, S. Kim, and B. M. Kwan, "Developing accurate longdistance 6-dof motion detection with one-dimensional laser sensors: Three-beam detection system," IEEE Tran. Ind. Electron., vol. 60, no. 8, pp. 3386-3395, Aug. 2013.

[11] AnkitLodha, Clinical Analytics - Transforming Clinical Development through Big Data, Vol-2, Issue-10, 2016

[12] AnkitLodha, Agile: Open Innovation to Revolutionize Pharmaceutical Strategy, Vol-2, Issue-12, 2016

[13] AnkitLodha, Analytics: An Intelligent Approach in Clinical Trail Management, Volume 6, Issue 5 , 1000e124 